This manuscript has been published by Elsevier in International Journal of Nursing Studies and can be found here: https://doi.org/10.1016/j.ijnurstu.2020.103552

Reference: Deschodt M, Laurent G, Cornelissen L, Yip O, Zúñiga F, Denhaerynck K, Briel M, Karabegovic A, De Geest S, and the INSPIRE consortium. Core components and impact of nurse-led integrated care models for home-dwelling older adults: a systematic review and metaanalysis. International Journal of Nursing Studies. 2020, 105.

\title{
Core components and impact of nurse-led integrated care models for home-dwelling older people: a systematic review and meta- analysis
}

Mieke Deschodt ${ }^{1,2}$, Gwen Laurent ${ }^{1}$, Lonne Cornelissen ${ }^{2}$, Olivia Yip ${ }^{1}$, Franziska Zúñiga ${ }^{1}$, Kris Denhaerynck $^{1}$, Matthias Briel ${ }^{3,4}$, Azra Karabegovic ${ }^{5}$, Sabina De Geest ${ }^{1,6}$, and the INSPIRE consortium $^{7}$

\section{Affiliations}

${ }^{1}$ University of Basel, Department Public Health, Nursing Science, Switzerland - Bernoullistrasse 28, 4056 Basel, Switzerland

2 KU Leuven, Department of Chronic Diseases, Metabolism and Ageing, Belgium, Herestraat 49 ON1 box 7073000 Leuven, Belgium

${ }^{3}$ McMaster University, Department of Health Research Methods, Evidence, and Impact, Canada 1280 Main Street West, Hamilton, Ontario, Canada

${ }^{4}$ University of Basel, Department Clinical Research, Switzerland - University Hospital Basel Schanzenstrasse 55, 4031 Basel, Switzerland

${ }^{5}$ Spitex Zürich Limmat AG · Fachentwicklung Chronic Care Kompetenz-Zentrum Spitex Zürich . Rotbuchstrasse $46 \cdot 8037$ Zürich

${ }^{6}$ KU Leuven, Academic Center for Nursing and Midwifery, Department of Public Health and Primary Care, Belgium - Kapucijnenvoer 35 blok d - box 7001, 3000 Leuven, Belgium

${ }^{7}$ Eva Blozik, Helsana-Gruppe Zürich; Rachel Jenkins, Spitex Zürich Limmat AG, Zürich; Nicole Probst-Hensch, Swiss Tropical and Public Health Institute, Basel, Switzerland; Carlos Quinto, Arztegeselleschaft Baselland; Matthias Schwenkglenks, Basel Pharmacoepidemiology Unit, Department of Pharmaceutical Sciences, University of Basel, Switzerland; Samuel Stenz, Nursing Science; Department Public Health, University of Basel, Switzerland; Penelope Vounatsou, Swiss Tropical and Public Health Institute, Basel, Switzerland; Andreas Zeller, Center for Primary Health Care, University of Basel, Basel, Switzerland; Leah Zullig, Department of Population Health Sciences, Duke University School of Medicine, Durham, USA.

Corresponding author: Mieke Deschodt, Nursing Science (INS), Department Public Health (DPH), Faculty of Medicine, University of Basel, Bernoullistrasse 28, CH-4056 Basel, Switzerland, Phone: $\underline{+41}$ $\underline{612070919}$. E-mail: mieke.deschodt@unibas.ch. Twitter: @mieke_deschodt 


\section{ABSTRACT}

BACKGROUND: Integrated care models are highly recommended to overcome care fragmentation in the multimorbid older population. Nurses are potentially ideally situated to fulfil the role as care coordinator to guide integrated care. No systematic review has been conducted specifically focusing on the impact of nurse-led integrated care models for older people in community settings.

OBJECTIVES: To identify core components of nurse-led integrated care models for the home-dwelling older population; to describe patient, service and process outcomes; and to evaluate the impact of these care models on quality of life, activities of daily living, hospitalisation, emergency department visits, nursing home admissions and mortality.

DESIGN: Systematic review and meta-analysis.

DATA SOURCES: English, Dutch, French, German and Spanish articles selected from PubMed and CINAHL, hand-search of reference lists of the included articles and grey literature.

REVIEW METHODS: A systematic search was conducted to identify prospective experimental or quasiexperimental studies detailing nurse-led integrated care models in the older home-dwelling population. Study characteristics and reported outcomes were tabulated. The core components of the models were mapped using the Sustainable intEgrated chronic care modeLs for multi-morbidity: delivery, FInancing, and performancE (SELFIE) framework. A random effects meta-analysis was conducted to study the overall effectiveness of the included care models on health-related quality of life, activities of daily living, hospitalisation, emergency department visits, nursing home admissions or mortality. Risk of bias was appraised using the revised Cochrane risk-of-bias tool for randomized trials and ROBINS-I tool for nonrandomized studies.

RESULTS: Nineteen studies were included studying a total of 22,168 patients. Core components of integrated care for multimorbid patients such as the involvement of a multidisciplinary team, high risk screening, tailored holistic assessment and an individualized care plan, were performed in a vast majority of the studies; however variability was observed in their operationalisation. Twenty-seven different patient, provider and service outcomes were reported, ranging from 1 to 13 per study. The meta-analyses could not demonstrate a beneficial impact on any of the predefined outcomes. Most included studies were of high risk for several biases.

CONCLUSION: The summarized evidence on nurse-led integrated care models in home-dwelling older people is inconclusive and of low quality. Future studies should include key components of implementation research, such as context analyses, process evaluations and proximal outcomes, to strengthen the evidence-base of nurse-led integrated care.

KEYWORDS: Activities of Daily Living; Community Health Nursing; Delivery of health care, integrated; Frail Elderly; Health Services for the Aged; Meta-analysis; Patient-centered Care; Quality of Life. 


\section{Contribution of the paper}

\section{What is already known about the topic?}

- Implementation of integrated care models is needed to provide person-centred care in the home-dwelling, multimorbid older population to avoid care fragmentation and negative health outcomes.

- Integrated care requires collaboration between health and social care providers to address the individual's multiple care problems, which are identified by performing a comprehensive geriatric assessment and are integrated in a tailored care plan.

- A team of multidisciplinary health care providers should be led by a coordinator, which could be a nurse, so that the care provided is coordinated, tailored and person-centered.

\section{What this paper adds}

- A large majority of core components deemed necessary in effective integrated care models for multimorbid people were included in the nurse-led integrated care models, except for those relating to financial aspects or technological support systems.

- Although individual studies demonstrated impact of nurse-led integrated care on quality of life, hospital admissions, emergency department visits and mortality, aggregated findings could not demonstrate a significant impact.

- Evidence-based implementation theories, context analysis and process evaluations are rarely used in the development and evaluation of nurse-led integrated care models, yet highly important to identify and overcome implementation problems that could result in intervention failure. 


\section{INTRODUCTION}

The prevalence of multimorbidity, defined as the co-occurrence of at least two chronic health conditions in one person, is rising. The Survey of Health, Ageing and Retirement in Europe indicated that in 2015 $41.5 \%$ of the population had multimorbidity [1]. People with multimorbidity have a lower life expectancy, a reduced quality of life, impaired functional status, poor physical and mental health and higher health care utilization [2]. Therefore, they often receive support from a large number of health and social care providers, and are subject to care fragmentation as a result of poor coordination of services and a lack of communication between all care providers involved [3]. Consequently, multimorbid people risk gaps in care delivery or duplication of services, leading to medication errors, ineffective treatment, contradictory recommendations, confusion and stress among patients and family, and higher health care costs [4-6]. With multimorbidity as well as cognitive and functional disabilities being more prevalent in older people, and the majority of older people preferring to age in their own house and community regardless of functional dependencies [7], caring for older people in the home- and community-care setting has become more complex $[8,9]$.

To address the complex needs of the older population, implementation of integrated care models has been recommended by the World Health Organisation (WHO), the National Institute for Health and Care Excellence and the King's Fund, among others [3, 10-13]. Integrated care has been described as a person-centred model of care that is structured to support coordinated, pro-active care led by a multidisciplinary core team and a lead coordinator communicating and cooperating across and within health sectors [4]. Integrated care interventions are complex interventions, with multiple interacting elements (i.e. different health care providers) and multiple levels targeted (i.e. organisational level or patient-level) [14]. In 2018, Leijten et al. reviewed the literature to determine a taxonomy of core concepts of integrated care that were considered relevant to provide integrated care to a multi-morbid population. This resulted in the Sustainable intEgrated chronic care modeLs for multi-morbidity: delivery, FInancing, and performancE (SELFIE) framework [15]. Each core concept was categorised in micro-, meso- and macro-levels for each of the six domains suggested by the WHO for a well-functioning health system, i.e., delivery of services, leadership, workforce, financing, information and research, and technology [16]. The aim of the SELFIE framework is to support the development, implementation and evaluation of integrated care programs for multi-morbid populations [15].

Care coordination by a named lead health care professional is considered one of the crucial elements in integrated care delivery $[11,15,17]$. Nurses are increasingly put forward as lead coordinators, because they often are people's first or only contact with health care professionals, are part of the local community and are holistically focused in their core activities [18-20]. The OECD has called upon advancing the role of nurses in primary care by introducing new complementary roles, such as nurse case managers or care coordinators [21]. However, no systematic review has been conducted to date to describe core concepts, outcomes and impact of nurse-led care integrated care models for community care. In 2016, Smith et al. evaluated the impact of interventions in patients with multimorbidity in primary and community settings and found little to no difference in medical outcomes and health services use, a modest reduction in depression scores, and a slight improvement in medication adherence and 
patient-related health behaviours. However, they did not specifically focus on nurse-led models of care in the older population [22]. Mullins et al. (2016) performed a review focusing on community nurse-led and nurse-involved models of care in which the importance of evaluating the role and activities of the nurse within these care models and the impact on health outcomes was specifically stressed [23]. However, this scoping review only included US-based models of care and the aim was limited to describing the models without focusing on the effectiveness of the models. The aim of this systematic review was therefore to:

1) describe the core components of nurse-led integrated care models in home-dwelling older populations and map them according to the SELFIE framework;

2) list the patient, provider and service outcomes measured in the included studies of nurse-led integrated care models; and

3) evaluate the overall effectiveness of nurse-led integrated care models for home-dwelling older people on health-related quality of life (HR-QoL), activities of daily living (ADL), hospital admissions, emergency department (ED) visits, nursing home admissions, and mortality compared to usual care.

\section{METHODOLOGY}

The review protocol was registered in the PROSPERO database (CRD42018093679). The review process was reported using the PRISMA guidelines [24].

\section{Search strategy}

A three-fold search strategy was performed. First, the electronic databases PubMed and CINAHL were searched for relevant papers using a search string that was limited to English, Dutch, French, German and Spanish papers published between $1^{\text {st }}$ January 2000 and $31^{\text {st }}$ December 2018. A final update was performed in June 2019. (Supplementary Materials. Table 1) Second, a hand-search was undertaken by checking the reference lists of the included papers and relevant reviews and by citation searching in PubMed, in which articles that cited an included article were screened. Third, OpenSIGLE (http://www.opengrey.eu/) was searched to identify grey literature, while Google Scholar was used to identify additional papers on care models described in the included papers.

\section{Eligibility and study selection}

All randomized controlled trials (RCTs) and prospective quasi-experimental studies in older adults aged $\geq 65$ or a reported mean age of $\geq 75$ years, living at home or in a service flat (flat with domestic service) were included. Studies needed to report the impact of a nurse-led integrated care model (i.e., a nurse was assessing needs and coordinating the care) on at least one of the following outcomes: HR-QoL, $A D L$, hospital admissions, ED visits, nursing home admissions, or mortality. Studies were excluded if they 1) had a qualitative or retrospective design; 2) included a disease-specific population, e.g., only focusing on patients with diabetes or patients in a palliative or end-of-life phase; 3) described models of care initiated in the hospital as part of outreach activities to improve the transition between hospital and home; or 4) described nurse-involved care models (i.e. a nurse was not the lead coordinator). 
After removing duplicate publications from the compiled Endnote database, the publications were screened against the in- and exclusion criteria based on title and abstract by at least one reviewer (GL, LC). Thereafter, two reviewers (GL, LC) independently screened the full-texts of the remaining publications. In case of disagreement, a third reviewer (MD) was consulted to discuss study inclusion until consensus was reached.

\section{Data extraction, risk of bias and evidence synthesis}

Data extraction forms were drafted and pilot-tested by two reviewers (LC, MD) using three of the included studies. Adjustments were made as needed. The data from the included studies were extracted and verified by at least two out of six reviewers (MD, GL, LC, KD, AK, RJ,). Study characteristics are summarized in table 1. The SELFIE framework from Leijten et al. (2018) was used to map the interventions described in the integrated care models in table 2 [15]. Patient, provider and service outcomes are tabulated in table 3. Risk of bias of randomized controlled trials (RCTs) and cluster RCTs was evaluated at the study level with the revised Cochrane risk of bias tool which grades the risk of selection, performance, attrition, detection and reporting bias [25], while risk of bias of the nonrandomized studies was evaluated with the ROBINS-I tool [26] (Supplementary material Table 2).

Meta-analyses were performed for HR-QoL (measured using the EQ-5D or RAND Short Form health questionnaire), ADL, hospital admissions, ED visits, nursing home admission, and mortality using the data available from the last follow-up point in each study. Stratification between randomised and nonrandomised studies was made. Effect sizes (i.e., standardized mean differences or odds ratio) were calculated, facilitated by an online calculator [27], and afterwards pooled by random-intercepts modelling to estimate between-study variability. Analyses were performed in SAS (SAS Institute, Cary, NC).

\section{RESULTS}

\section{Study selection}

Through the systematic search, 2834 potentially relevant articles were identified. After removing duplicates, screening the titles and abstracts, screening reference lists of included articles and additional searches, 124 full-texts were considered for inclusion. A total of 103 articles were excluded, resulting in a final number of 21 included papers, based on 19 studies [28-48] (Supplementary Material Figure 1). Seven protocol papers provided additional information regarding the methodology of the study or the content of the integrated care models [49-55]. 
Table 1: Study characteristics

\begin{tabular}{|c|c|c|c|c|c|c|}
\hline Study & Name of Intervention & Country & $\begin{array}{l}\text { Inclusion } \\
\text { period }\end{array}$ & Design & Inclusion and screening criteria & Sample \\
\hline Boult 2011 & Guided care & US & $2006-2008$ & $\begin{array}{l}\text { Cluster-randomized } \\
\text { controlled trial }\end{array}$ & $\begin{array}{l}\geq 65 \text { years } \\
\text { High risk of high health services use in next year ( } \leq 6 \\
\text { self-reported health status) }\end{array}$ & $\begin{array}{l}\text { IG: } 446 \\
\text { CG: } 404\end{array}$ \\
\hline $\begin{array}{l}\text { Bouman } \\
2008\end{array}$ & Home Visiting Program & Netherlands & 2003-2004 & $\begin{array}{l}\text { Randomized } \\
\text { controlled trial }\end{array}$ & $\begin{array}{l}70-84 \text { years } \\
\text { Poor health status }(\text { EasyCare }<6)\end{array}$ & $\begin{array}{l}\text { IG: } 160 \\
\text { CG: } 170\end{array}$ \\
\hline $\begin{array}{l}\text { Counsell } \\
2007\end{array}$ & $\begin{array}{l}\text { Geriatric Resources for } \\
\text { Assessment and Care } \\
\text { of Elders (GRACE) }\end{array}$ & US & $2002-2004$ & $\begin{array}{l}\text { Cluster-randomized } \\
\text { controlled trial }\end{array}$ & $\begin{array}{l}\geq 65 \text { years } \\
\text { Income }<200 \% \text { of the federal poverty level } \\
\geq 1 \text { primary care clinician visit in the past year }\end{array}$ & $\begin{array}{l}\text { IG: } 474 \\
\text { CG: } 477\end{array}$ \\
\hline Dorr 2008 & Care Management Plus & US & $2002-2005$ & $\begin{array}{l}\text { Non-randomized } \\
\text { controlled trial }\end{array}$ & $\begin{array}{l}\geq 65 \text { years } \\
\geq 11 \text { months enrolment in Medicare Part B in the 1- } \\
\text { year period before the enrolment date } \\
\text { Depression (Patient Health Questionnaire } 9 \text { ) }\end{array}$ & $\begin{array}{l}\text { IG: } 1144 \\
\text { CG: } 2288\end{array}$ \\
\hline $\begin{array}{l}\text { Gravelle } \\
2007\end{array}$ & Evercare & England & 2003-2005 & $\begin{array}{l}\text { Before-and-after } \\
\text { study }\end{array}$ & $\begin{array}{l}\geq 65 \text { years } \\
\geq 2 \text { emergency admissions in preceding } 13 \text { months }\end{array}$ & $\begin{array}{l}\text { Intervention } \\
\text { practices: } 64\end{array}$ \\
\hline $\begin{array}{l}\text { Hoogendijk } \\
2016\end{array}$ & $\begin{array}{l}\text { Geriatric Care model } \\
(\mathrm{GCM})\end{array}$ & Netherlands & $2010-2011$ & $\begin{array}{l}\text { Stepped wedge } \\
\text { cluster-randomized } \\
\text { controlled trial }\end{array}$ & $\begin{array}{l}\geq 65 \text { years } \\
\text { PRISMA-7 score } \geq 3\end{array}$ & $\begin{array}{l}\text { Group 1: } 456 \\
\text { Group 2: } 227 \\
\text { Group 3: } 238 \\
\text { Group 4: } 226\end{array}$ \\
\hline Imhof 2012 & $\begin{array}{l}\text { Health Consultation } \\
\text { Program }\end{array}$ & Switzerland & $2008-2011$ & $\begin{array}{l}\text { Randomized } \\
\text { controlled trial }\end{array}$ & $\begin{array}{l}\geq 80 \text { years } \\
\text { Cognitive intact according to Clinical Dementia } \\
\text { Rating Scale }\end{array}$ & $\begin{array}{l}\text { IG: } 231 \\
\text { CG: } 230\end{array}$ \\
\hline King 2018 & $\begin{array}{l}\text { Gerontology Nurse } \\
\text { specialist }\end{array}$ & $\begin{array}{l}\text { New } \\
\text { Zealand }\end{array}$ & $2009-2013$ & $\begin{array}{l}\text { Controlled before- } \\
\text { and-after study }\end{array}$ & $\begin{array}{l}\geq 75 \text { years } \\
\text { Enrolled in one of two primary healthcare practices } \\
\text { Brief Risk Identification for Geriatric Health Tool }\end{array}$ & $\begin{array}{l}\text { IG: } 517 \\
\text { CG: } 883\end{array}$ \\
\hline $\begin{array}{l}\text { Looman } \\
2014,2016\end{array}$ & $\begin{array}{l}\text { Walcheren Integrated } \\
\text { Care Model }\end{array}$ & Netherlands & $2010-2011$ & $\begin{array}{l}\text { Controlled before- } \\
\text { and-after study }\end{array}$ & $\begin{array}{l}\geq 75 \text { years } \\
\text { Frail according to Groningen Frailty Indicator, not on } \\
\text { waiting list for nursing home admission, life } \\
\text { expectancy of }>6 \text { months }\end{array}$ & $\begin{array}{l}\text { IG: } 184 \\
\text { CG: } 193\end{array}$ \\
\hline $\begin{array}{l}\text { Markle-Reid } \\
2006\end{array}$ & $\begin{array}{l}\text { Proactive nursing } \\
\text { health promotion }\end{array}$ & Canada & 2001-2002 & $\begin{array}{l}\text { Randomized } \\
\text { controlled trial }\end{array}$ & $\begin{array}{l}\geq 75 \text { years } \\
\text { Newly referred to support services through the } \\
\text { Community Care Access Centre (CCAC) } \\
\text { Need for assistance with personal care }\end{array}$ & $\begin{array}{l}\text { IG: } 144 \\
\text { CG: } 144\end{array}$ \\
\hline Melis 2008 & $\begin{array}{l}\text { Dutch Geriatric } \\
\text { Intervention Program }\end{array}$ & Netherlands & 2003-2005 & $\begin{array}{l}\text { Pseudo cluster- } \\
\text { randomized } \\
\text { controlled trial }\end{array}$ & $\begin{array}{l}\geq 70 \text { years } \\
\text { Limitations in cognition (MMSE } \leq 26 \text { ), (instrumental), } \\
\text { ADL (GARS } 3 \geq 25 \text { ) or mental wellbeing and MOS } \\
\text { mental health } \leq 75\end{array}$ & $\begin{array}{l}\text { IG: } 85 \\
\text { CG: } 66\end{array}$ \\
\hline
\end{tabular}




\begin{tabular}{|c|c|c|c|c|c|c|}
\hline $\begin{array}{l}\text { Metzelthin } \\
2013\end{array}$ & Prevention of Care & Netherlands & $2009-2010$ & $\begin{array}{l}\text { Cluster-randomized } \\
\text { controlled trial }\end{array}$ & $\begin{array}{l}\geq 70 \text { years } \\
\text { Frail older patients (Groningen Frailty Indicator) } \\
\text { Exclusion of terminally ill patients, confined to bed }\end{array}$ & $\begin{array}{l}\text { IG: } 193 \\
\text { CG: } 153\end{array}$ \\
\hline $\begin{array}{l}\text { Morales- } \\
\text { Asencio } \\
2008\end{array}$ & l & Spain & 2003-2006 & $\begin{array}{l}\text { Non-randomized } \\
\text { controlled trial }\end{array}$ & $\begin{array}{l}\text { Terminally ill, requiring ADL assistance and are } \\
\text { immobilised at home, or recently discharged from } \\
\text { hospital + their main caregivers }\end{array}$ & $\begin{array}{l}\text { Patients: } \\
\text { IG: } 182, \text { CG: } 160 \\
\text { Caregivers: } \\
\text { IG: } 117, \text { CG: } 88\end{array}$ \\
\hline $\begin{array}{l}\text { Newcomer } \\
2004\end{array}$ & $\begin{array}{l}\text { Enhanced Case } \\
\text { Management }\end{array}$ & US & 2001-2003 & $\begin{array}{l}\text { Randomized } \\
\text { controlled trial }\end{array}$ & $\begin{array}{l}\geq 80 \text { years or } \geq 65 \text { years with chronic obstructive } \\
\text { pulmonary disease, congestive heart failure, coronary } \\
\text { disease or diabetes. Not in nursing home, } \\
\text { Alzheimer's facility or at hospices }\end{array}$ & $\begin{array}{l}\text { IG: } 1537 \\
\text { CG: } 1542\end{array}$ \\
\hline $\begin{array}{l}\text { Reckrey } \\
2015\end{array}$ & $\begin{array}{l}\text { Mount Sinai Visiting } \\
\text { Doctors }\end{array}$ & US & 2010-2011 & $\begin{array}{l}\text { Non-randomised } \\
\text { controlled trial }\end{array}$ & $\begin{array}{l}\text { Home bound (able to leave home only with great } \\
\text { difficulty and for infrequent or short absences) }\end{array}$ & $\begin{array}{l}\text { IG: } 347 \\
\text { CG: } 1074\end{array}$ \\
\hline Ruikes 2016 & CareWell & Netherlands & 2011-2012 & $\begin{array}{l}\text { Cluster non- } \\
\text { randomized } \\
\text { controlled trial }\end{array}$ & $\begin{array}{l}\geq 70 \text { years } \\
\text { Frail according to EASY-Care Two-Step Older } \\
\text { Persons Screening instrument and clinical } \\
\text { judgement, no terminal illness }\end{array}$ & $\begin{array}{l}\text { IG: } 287 \\
\text { CG: } 249\end{array}$ \\
\hline $\begin{array}{l}\text { Ryvicker } \\
2011\end{array}$ & $\begin{array}{l}\text { Home Health Aids } \\
\text { (HHA) Partnering } \\
\text { Collaborative }\end{array}$ & US & $2005-2006$ & $\begin{array}{l}\text { Cluster-randomized } \\
\text { controlled trial }\end{array}$ & $\begin{array}{l}\text { Patients: Min. } 1 \text { HHA visit last year, life expectancy > } \\
6 \text { months, room for improvement in min. } 1 \text { ADL } \\
\text { Teams: Visiting Nurse Service of Bronx, Brooklyn, } \\
\text { Manhattan and Queens, NY }\end{array}$ & $\begin{array}{l}\text { IG: } 22 \text { teams, } \\
\text { 1516 patients } \\
\text { CG: } 23 \text { teams, } \\
\text { 1774 patients }\end{array}$ \\
\hline $\begin{array}{l}\text { Stijnen } \\
2015 a \\
2015 b\end{array}$ & $\begin{array}{l}\text { Getting OLD the } \\
\text { healthy way }\end{array}$ & Netherlands & 2010-2011 & $\begin{array}{l}\text { Cluster non- } \\
\text { randomized } \\
\text { controlled trial }\end{array}$ & $\begin{array}{l}\geq 75 \text { years } \\
\text { Not on waiting list for nursing home admission, no } \\
\text { close medical supervision and not terminally ill }\end{array}$ & $\begin{array}{l}\text { IG: } 586 \\
\text { CG: } 941\end{array}$ \\
\hline Suijker 2016 & $\begin{array}{l}\text { Nurse-led multifactorial } \\
\text { care }\end{array}$ & Netherlands & 2010 & $\begin{array}{l}\text { Cluster-randomized } \\
\text { controlled trial }\end{array}$ & $\begin{array}{l}\geq 70 \text { years } \\
\text { At increased risk of functional decline (ISAR - Primary } \\
\text { Care } \geq 2 \text { ), life expectancy } \geq 3 \text { month }\end{array}$ & $\begin{array}{l}\text { IG: } 1209 \\
\text { CG: } 1074\end{array}$ \\
\hline
\end{tabular}




\section{Characteristics of the included studies}

Six cluster RCTs, five RCTs and eight non-randomized studies were included, of which eleven were conducted in Europe, seven in North America and one in New Zealand (Table 1). The sample size ranged from 151 to 3,432 participants with the mean age of intervention patients varying from 71.8 to 85 years. Study follow-up ranged from three to 24 months. Overall, the included randomized studies were at considerable risk of bias and findings should be interpreted in that perspective (Supplementary Materials Table 2).

\section{Description of the care models}

The core components described in the included care models were summarised using the six domains and concepts at micro-level of the SELFIE framework from Leijten et al. (2018) [15] (Table 2).

\section{Service delivery}

In all but one model $(\mathrm{n}=18)$, a person-centered care approach by means of a tailored holistic assessment or comprehensive geriatric assessment was performed. In nine studies, older people were encouraged by the care team to improve their self-management abilities by providing them advice $(n=3)$; improving knowledge and establishing a positive attitude towards change $(n=2)$; motivational interviewing $(n=1)$; or assessing self-efficacy $(n=1)$. Informal caregivers were involved in the decision-making process in 13 studies. Attention for treatment and guideline interactions in view of polypharmacy and multimorbidity was addressed in ten studies, while a clear focus on continuity of care was reported in 16 studies. Follow-up of care was described in all studies and was performed by conducting home visits $(n=18)$, telephone calls ( $n$ $=10)$ or re-assessment $(n=2)$ of the older person.

\section{Leadership and governance}

In most studies, shared decision-making was described as an integral part of the integrated care model (n $=14$ ) and a named care coordinator set up an individual care plan to respond to the individuals' needs and preferences $(n=16)$. In complex cases, a case manager was assigned to tailor care processes $(n=10)$. 
Table 2: Core concepts of the care models of the included studies according to the micro level of the Sustainable intEgrated chronic care modeL for multimorbidity: delivery, FInancing, and performance (SELFIE) framework.

\begin{tabular}{|c|c|c|c|c|c|c|c|c|c|c|c|c|c|c|c|c|c|c|c|c|c|}
\hline \multirow[t]{2}{*}{ Study } & \multicolumn{7}{|c|}{ Service delivery } & \multicolumn{3}{|c|}{$\begin{array}{c}\text { Leadership \& } \\
\text { governance }\end{array}$} & \multicolumn{4}{|c|}{ Workforce } & \multicolumn{3}{|c|}{ Financing } & \multicolumn{2}{|c|}{ Technology } & \multicolumn{2}{|c|}{$\begin{array}{c}\text { Information \& } \\
\text { research }\end{array}$} \\
\hline & $\begin{array}{l}\bar{d} \\
d \\
d \\
\bar{d} \\
0 \\
1 \\
0 \\
0 \\
\frac{w}{d} \\
0\end{array}$ & 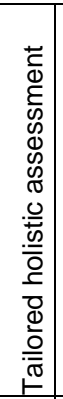 & 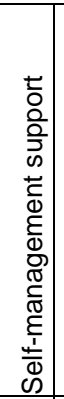 & 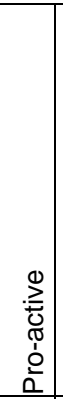 & 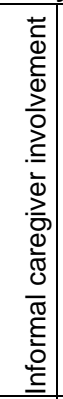 & 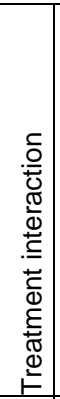 & 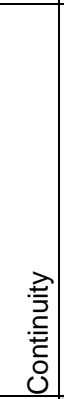 & 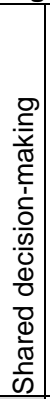 & 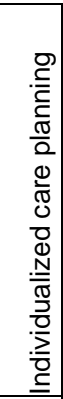 & 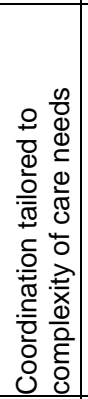 & 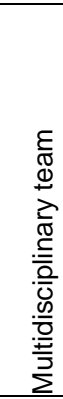 & 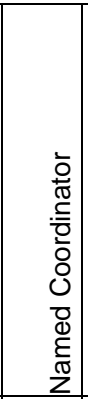 & $\begin{array}{l}0 \\
0 \\
0 \\
0 \\
0 \\
0 \\
0\end{array}$ & 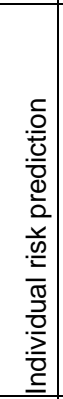 & 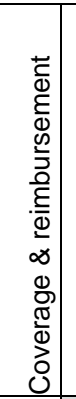 & 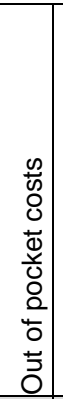 & 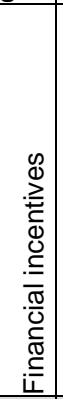 & 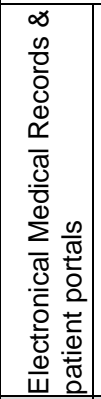 & 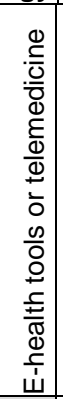 & 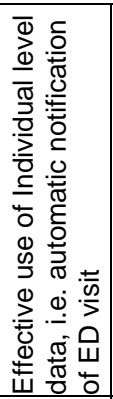 & 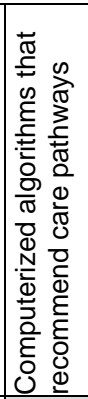 \\
\hline Boult 2011 & $x$ & $x$ & $\mathrm{x}$ & $x$ & $x$ & $x$ & $\mathrm{x}$ & & $x$ & $x$ & $x$ & $\mathrm{RN}$ & $\mathrm{RN}, \mathrm{GP}$ & $x$ & $x$ & & & & & $x$ & \\
\hline Bouman 2008 & $x$ & $x$ & $x$ & $x$ & & & $x$ & $x$ & $x$ & & $x$ & $\mathrm{RN}$ & RN, GP & $x$ & & & & & & & \\
\hline Counsell 2007 & $x$ & $x$ & $x$ & $x$ & $x$ & $x$ & $x$ & $x$ & $x$ & $x$ & $x$ & APN & APN, GP & & & & & $x$ & $x$ & $x$ & \\
\hline Dorr 2008 & $x$ & $x$ & $x$ & $x$ & $x$ & & $x$ & $x$ & $x$ & $x$ & $x$ & $\mathrm{RN}$ & $\mathrm{RN}, \mathrm{GP}$ & $x$ & & & & $x$ & $x$ & $x$ & \\
\hline Gravelle 2007 & $x$ & $\mathrm{x}$ & & $x$ & & & $x$ & $x$ & $x$ & & $x$ & APN & APN, GP & $x$ & & & & & & $x$ & \\
\hline Hoogendijk 2016 & $x$ & $x$ & & $x$ & $x$ & & $x$ & $x$ & $x$ & & $x$ & PN & $\mathrm{PN}, \mathrm{GP}$ & $x$ & & & & & & & \\
\hline Imhof 2012 & $x$ & $x$ & $x$ & $x$ & $x$ & $\mathrm{x}$ & $x$ & $x$ & $x$ & $x$ & $x$ & APN & APN, GP & & & & & & & $\mathrm{x}$ & \\
\hline King 2018 & $\mathrm{x}$ & $\mathrm{x}$ & & $x$ & & $\mathrm{x}$ & & $x$ & $x$ & & $\mathrm{x}$ & GNS & & $\mathrm{x}$ & & & & $x$ & & $\mathrm{x}$ & \\
\hline Looman 2014;2016 & $x$ & $\mathrm{x}$ & & $x$ & $x$ & & $x$ & $x$ & $x$ & $x$ & $x$ & & $\mathrm{NP}, \mathrm{GP}$ & $x$ & & & & & & & \\
\hline Markle-Reid 2006 & $x$ & $\mathrm{x}$ & $x$ & $\mathrm{x}$ & $x$ & $x$ & & & $x$ & & $x$ & $\mathrm{RN}$ & & $x$ & & $x$ & & & & & \\
\hline Melis 2008 & $x$ & $x$ & $x$ & $x$ & & $x$ & $x$ & $x$ & $x$ & $x$ & $x$ & GNS & GNS, GP & $x$ & & & & & & & \\
\hline Metzelthin 2013 & $x$ & $x$ & $x$ & $x$ & $x$ & $x$ & $x$ & $x$ & $x$ & $x$ & $x$ & $\mathrm{PN}$ & PN, GP & $x$ & & & & & & & \\
\hline Morales-Asencio 2008 & $\mathrm{x}$ & $\mathrm{x}$ & & $x$ & $\mathrm{x}$ & & $\mathrm{x}$ & $\mathrm{x}$ & & $\mathrm{x}$ & $x$ & RN & RN, GP & & & & & & & & \\
\hline Newcomer 2004 & $\mathrm{x}$ & $x$ & & $\mathrm{x}$ & $\mathrm{x}$ & $\mathrm{x}$ & & & $x$ & $\mathrm{x}$ & $x$ & RN & & & $x$ & & & $\mathrm{x}$ & & $\mathrm{x}$ & \\
\hline Reckrey 2015 & $x$ & $x$ & & & $x$ & $\mathrm{x}$ & $\mathrm{x}$ & $\mathrm{x}$ & $x$ & $\mathrm{x}$ & $\mathrm{x}$ & NP & NP, GP, SW & & & & & $x$ & & & \\
\hline Ruikes 2016 & $x$ & & & $\mathrm{x}$ & & $\mathrm{x}$ & $x$ & $x$ & $x$ & $x$ & $x$ & $\mathrm{CM}$ & $\begin{array}{c}\text { PN, GP, ECP, } \\
\text { SW }\end{array}$ & $x$ & & & & $\mathrm{x}$ & & $x$ & \\
\hline Ryvicker 2011 & & $x$ & & $x$ & $x$ & & $x$ & $x$ & & & $x$ & RN & RN, SW & & & & & & & & \\
\hline Stijnen 2015a, 2015b & $x$ & $x$ & & $x$ & & & $x$ & $x$ & $x$ & & $x$ & PN & PN, GP & & & & & & & & \\
\hline Suijker 2016 & $x$ & $x$ & $\mathrm{x}$ & $x$ & $\mathrm{x}$ & & $x$ & & $x$ & & $x$ & $\mathrm{RN}$ & $\mathrm{RN}, \mathrm{GP}$ & $x$ & & & & & & & \\
\hline
\end{tabular}

$\mathbf{A P N}=$ advanced practice nurse; $\mathbf{C M}=$ case manager; $\mathbf{E C P}=$ elderly care physician; $\mathbf{G N S}=$ geriatric nurse specialist; $\mathbf{G P}=$ general practitioner;

$\mathbf{N P}=$ nurse practitioner; $\mathbf{P N}=$ practice nurse; $\mathbf{R N}=$ registered nurse; $\mathbf{S} \boldsymbol{W}=$ social worker 


\section{Workforce}

In all care models, a multidisciplinary team was involved in patient care, with a nurse as named coordinator in eighteen studies. In sixteen studies, a differentiation was made between a core group of professionals and the wider multidisciplinary team that can be called upon. The core group is responsible for regular care contacts and relationship-building, whereas the larger multidisciplinary team will see the patient depending on identified needs and treatment goals. A practice nurse, registered nurse, advanced nurse practitioner or geriatric nurse specialist, together with the GP were part of this core group in thirteen studies. The social worker was part of the core group in two studies mainly to perform home visits or to address specific social service related problems for the patient. Referrals to a specialist or other health care services took place in fourteen studies [28, 30-35, 37, 40-44, 48]. Staff training was mentioned in nine studies [32, 34-37, 40, 43, $46,48]$. Individual risk prediction, which is considered important to determine which professionals need to be involved in care planning, was performed in the majority of the studies $(n=12)$ but the screening tool used was different in each study.

\section{Financing}

Boult et al. (2011) reported the study findings in relation to the patient's health insurance and reported the following three categories: 1) older persons insured by Kaiser-Permanente, a non-for-profit integrated model of health care delivery driven by the needs of members rather than stakeholders; 2 ) traditional feefor-service Medicare; and 3) the TRICARE/US Family Health Plan, a federal health insurance program for retired military personnel and their dependents. They found that guided care reduced health care services use to a greater extent in the Kaiser-Permanente subgroup [28]. No other studies reported information regarding coverage and reimbursement structures to ensure equity in financial access for those who need them. No studies reported using financial incentives to motivate persons with multimorbidity to participate in integrated care programmes.

\section{Technology}

Six studies mentioned the use of electronic medical records and three studies the use of web-based technology to support intervention delivery. No studies mentioned the use of telemedicine, assistive technologies or remote monitoring of clinical parameters.

\section{Information and research}

In eight studies, information regarding previous ED visits, details about hospital discharge and medicinerelated information was used in the treatment plan. No studies reported including computerized algorithms that recommend care pathways.

\section{Reported patient, provider and service outcomes}

Twenty-seven outcomes were measured with the number of outcomes ranging from 1 to 13 per study (Table 3). Mortality, HR-QoL, ADL, and hospital admissions were most often reported, while the provider outcomes, i.e., quality of care, caregiver burden and provider satisfaction, were measured in only one study, respectively. 
Table 3: Reported patient, provider and service outcomes

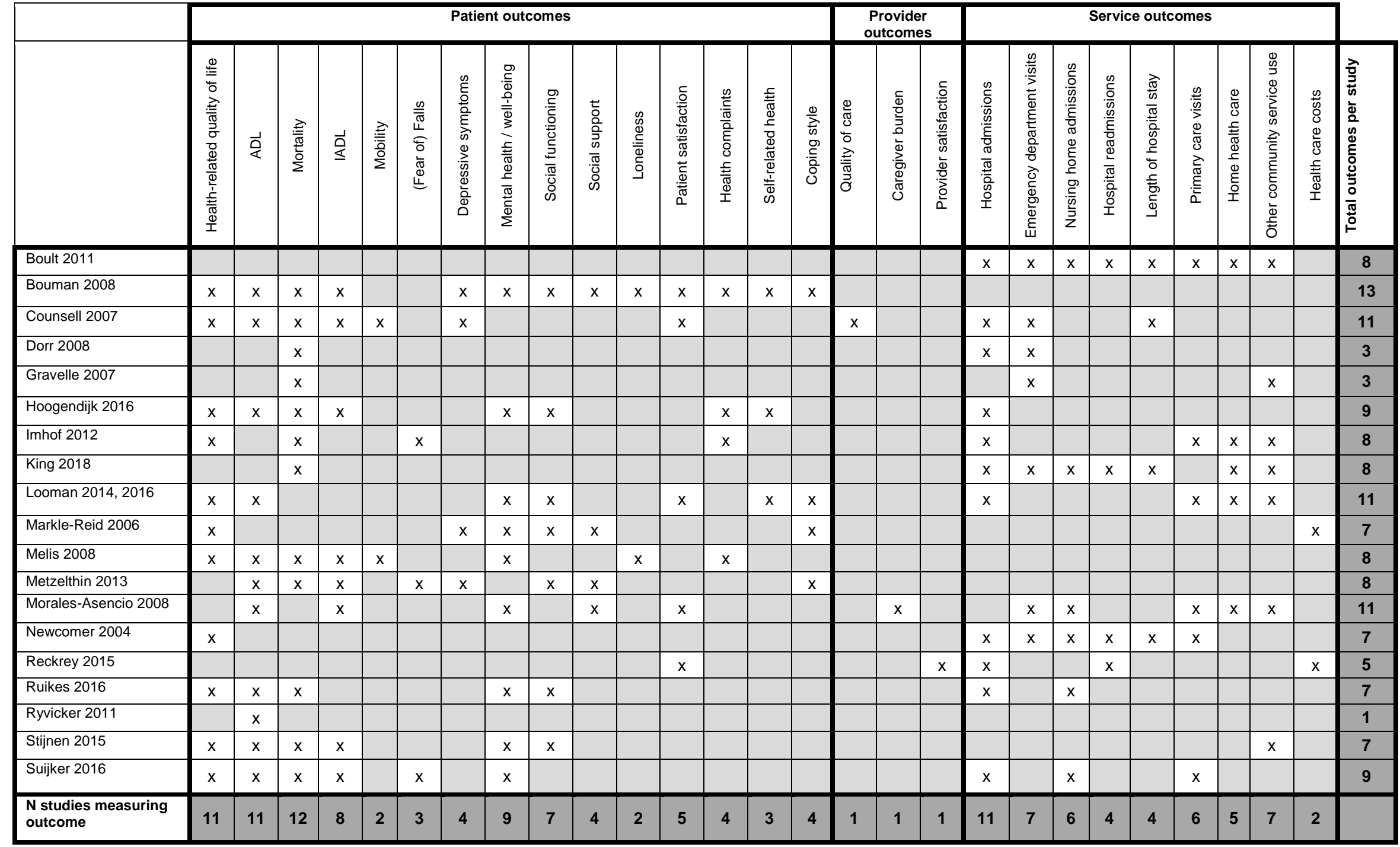




\section{Effect of nurse-led integrated care}

Random-effects pooled estimates of EQ-5D [29, 34, 38, 39, 48] and the RAND Short Form health questionnaire [29-31, 34, 40, 44] did not show an overall significant impact on health-related quality of life (Table 4). At individual study level, Suijker et al. (2016) reported a statistically significant improvement in quality of life (using the EQ-5D) after 18 months and 24 months, with a standardized mean difference of $0.13(95 \% \mathrm{Cl}: 0.03-0.23)$ and $0.10(95 \% \mathrm{Cl}: 0.01-0.20)$, respectively [48]. Both Bouman et al. (2008) and Counsel et al. (2007) reported better mental health scores using the RAND-36 in the intervention group with standardized mean differences of $0.24(95 \% \mathrm{Cl}: 0.01-0.47)$ and 0.21 (95\% Cl: $0.09-0.34$ ), respectively [30,31]. Stijnen et al. (2015) showed a significant impact on the social functioning subscale of the RAND-36 [37].

Random-effects pooled estimates of $A D L$ measured with either Katz $\operatorname{ADL}[29,34,48]$, the Groningen Activity Restriction Scale (GARS) [37, 41, 42], the Barthel Index [43] or similar ADL instruments [30, 31, 38,39 ] including a total of eleven studies did not show any significant results (Cohen's d $0.006,95 \% \mathrm{Cl}$ 0.041 to 0.054 ). Also at the individual study level, no significant impact was observed on ADL outcomes [29-31, 34, 37-39, 41-43, 46, 48]

Random-effects pooled estimates including data of eleven studies showed no significant impact on hospital admission rates (OR $0.944,95 \% \mathrm{Cl} 0.871$ to 1.022 ) [28-30, 32, 34-36, 38, 39, 44, 45, 48]. A significant reduction of hospital admissions was found in one individual trial. Imhof et al. (2012) reported a significantly lower percentage of hospital admissions in a 3-month period in the intervention group (23\%) compared to the control group $(33 \%)(\mathrm{OR}=0.69,95 \% \mathrm{Cl}: 0.47-0.97)$ [35].

Random-effects pooled estimates of ED visits including data of seven studies did not show a significant impact on ED visits (OR 1.001, 95\% Cl 0.844 to 1.187 ) [28, 30, 32, 33, 36, 43, 44]. Only one study reported a significantly lower number of cumulative ED visits in the intervention group after 2 years $(n=1445)$ compared to the control group $(n=1748 ;$ OR $=0.73,95 \% \mathrm{Cl}$ : $0.58-0.93)$ [30].

Random-effects pooled estimates did not show an overall significant impact on nursing home admissions (OR $0.907,95 \% \mathrm{Cl} 0.713$ to 1.155 ) [28, 29, 36, 43, 44, 48] or mortality (OR $0.946,95 \% \mathrm{Cl} 0.389-2.302$ ) [29-37, 41, 42, 48]. None of the six studies reporting nursing home admission rates found a significant impact of the intervention. One of the twelve studies reporting mortality rates found a significant decrease in favour of the intervention group from $9.2 \%$ to $6.5 \%(\mathrm{OR}=0.84,95 \% \mathrm{Cl}: 0.74-0.96)$ after one year follow-up. [32].

Meta-analyses were also done per outcome stratifying the randomized and non-randomized studies but this did not alter any of our findings (Table 4). 
Table 4: Pooled estimates on patient-relevant outcomes

\section{Outcome}

\begin{tabular}{|c|c|}
\hline \multirow[t]{3}{*}{ HR-QoL: EQ-5D } & All studies [29, 34, 38, 39, 48] \\
\hline & Randomized \\
\hline & Non-randomized \\
\hline \multirow[t]{3}{*}{ HR-QoL: RAND-SF } & All studies [29-31, 34, 40, 44] \\
\hline & Randomized \\
\hline & Non-randomized \\
\hline \multirow[t]{3}{*}{ Activities of daily living } & All studies [31, 34, 37-39, 42, 43, 48] \\
\hline & Randomized \\
\hline & Non-randomized \\
\hline \multirow[t]{3}{*}{ Hospital admissions } & All studies $[28,30,32,34-36,39,44,45,48]$ \\
\hline & Randomized \\
\hline & Non-randomized \\
\hline \multirow[t]{3}{*}{ ED visits } & All studies $[28,30,32,33,36,43,44]$ \\
\hline & Randomized \\
\hline & Non-randomized \\
\hline \multirow{3}{*}{$\begin{array}{l}\text { Nursing home } \\
\text { admission }\end{array}$} & All studies $[28,29,36,43,44,48]$ \\
\hline & Randomized \\
\hline & Non-randomized \\
\hline \multirow[t]{3}{*}{ Mortality } & All studies [29-37, 41, 42, 48] \\
\hline & Randomized \\
\hline & Non-randomized \\
\hline
\end{tabular}

\begin{tabular}{|c|c|c|c|c|}
\hline $\begin{array}{l}\mathrm{N} \text { of } \\
\text { tients }\end{array}$ & $\begin{array}{l}\text { Median (Q1 - } \\
\text { Q3) follow-up }\end{array}$ & $\begin{array}{c}\text { Estimate Cohen's d } \\
(95 \% \mathrm{Cl})\end{array}$ & $\mathrm{I}^{2}(95 \% \mathrm{Cl})$ & $\begin{array}{c}\text { Hetero- } \\
\text { geneity } \\
\text { (p-value) }\end{array}$ \\
\hline 7038 & $24(12-24)$ & $0.022(-0.055-0.098)$ & $0.0 \%(0.0-42.1)$ & 0.861 \\
\hline 6292 & $24(24-24)$ & $0.025(-0.299-0.348)$ & $0.0 \%(.-)$. & 0.519 \\
\hline 746 & $12(12-12)$ & $0.000(-0.933-0.934)$ & $0.0 \%(.-)$. & 0.625 \\
\hline 0339 & $24(12-24)$ & $0.010(-0.038-0.059)$ & $25.8 \%(0.0-67.7)$ & 0.232 \\
\hline 9962 & $24(12-24)$ & $0.003(-0.048-0.055)$ & $0.0 \%(0.0-73.8)$ & 0.441 \\
\hline 377 & $12(.-)$ & $0.194(-0.008-0.397)$ & Only one study & l \\
\hline 0249 & $21(12-24)$ & $0.006(-0.041-0.054)$ & $32.5 \%(0.0-67.9)$ & 0.148 \\
\hline 7989 & $24(24-24)$ & $-0.020(-0.078-0.038)$ & $17.0 \%(0.0-61.8)$ & 0.304 \\
\hline 2260 & $12(9-15)$ & $0.091(-0.048-0.231)$ & $0.0 \%(0.0-79.4)$ & 0.548 \\
\hline $\begin{array}{l}\mathrm{N} \text { of } \\
\text { tients }\end{array}$ & $\begin{array}{l}\text { Median (Q1 - } \\
\text { Q3) follow-up }\end{array}$ & $\begin{array}{c}\text { Estimate OR } \\
(95 \% \mathrm{Cl})\end{array}$ & $I^{2}(95 \% \mathrm{Cl})$ & \\
\hline 6942 & $12(12-24)$ & $0.944(0.871-1.022)$ & $4.7 \%(0.0-62.2)$ & 0.399 \\
\hline 9903 & $22(12-24)$ & $0.911(0.796-1.042)$ & $2.0 \%(0.0-75.3)$ & 0.404 \\
\hline 7039 & $20(12-24)$ & $0.980(0.834-1.151)$ & $11.5 \%(0.0-81.9)$ & 0.340 \\
\hline 6834 & $12(6-24)$ & $1.001(0.844-1.187)$ & $61.7 \%(12.5-83.3)$ & 0.016 \\
\hline 2455 & $12(.-)$. & $0.995(0.831-1.192)$ & Only one study & 1 \\
\hline 4379 & $16(6-24)$ & $1.004(0.805-1.252)$ & $67.9 \%(23.8-86.5)$ & 0.008 \\
\hline 5770 & $12(12-20)$ & $0.907(0.713-1.155)$ & $0.0 \%(0.0-44.1)$ & 0.814 \\
\hline 3538 & $20(12-24)$ & $0.898(0.571-1.412)$ & $0.0 \%(0.0-85.8)$ & 0.612 \\
\hline 2232 & $6(6-12)$ & $0.946(0.389-2.302)$ & $0.0 \%(0.0-88.5)$ & 0.544 \\
\hline 8760 & $18(9-24)$ & $0.946(0.389-2.302)$ & $29.3 \%(0.0-64.3)$ & 0.158 \\
\hline 4478 & $18(6-24)$ & $0.946(0.389-2.302)$ & $0.0 \%(0.0-70.0)$ & 0.445 \\
\hline 4282 & $12(12-18)$ & $0.946(0.389-2.302)$ & $57.9 \%(0.0-84.4)$ & 0.050 \\
\hline
\end{tabular}




\section{Discussion}

Given the increasing care complexity of older people living at home and their preference to age in place, there is consensus among leading international institutions towards further investment in integrated care models for frail older people. In this systematic review focusing on nurse-led integrated care models, 19 studies were included measuring a total of 27 different outcomes. Individual studies demonstrated an impact on mortality [32], HR-QoL [30, 31, 48], hospital admissions [35] and ED visits [30], but no overall effect was found in the meta-analyses on any of the outcomes.

Although this is the first systematic review describing core concepts and effectiveness of nurse-led integrated care models, other reviews were conducted focusing on related care models for the homedwelling older and/or frail population [56-59]. Two meta-analyses of RCTs studying preventive primary care outreach interventions in 19 RCTs [57] and multidimensional preventive home visit programs in 21 RCTs [59] respectively showed a beneficial effect on mortality rate and likelihood to continue living in the community, but not on nursing home admissions, hospitalisation and functional status. Hopman et al. (2016) reported indications of increased quality of care, better HR-QoL and lower depression scores in experimental studies of any design evaluating comprehensive care programs for patients with multiple chronic conditions or frailty of any age, but concluded that evidence remains insufficient due to the lack of good-quality studies [56]. The systematic review of Looman et al. (2019) including 29 controlled studies demonstrated no effect of preventive, integrated care on the majority of the reported outcomes, except for seldom-reported outcomes such as well-being. Most promising was the improvement of care processes for preventive, integrated care interventions as compared to usual care [58].

Hence, despite the clear calls from leading institutions to implement integrated care models, the current evidence base is not as conclusive as one might expect, except for some individual studies reporting a beneficial impact on several outcomes and better patient and provider experiences due to better care organisation, coordination and collaboration in integrated care models [60-62]. One of the main challenges is that integrated care models are complex interventions; a complexity that can be observed in terms of the number of and interactions between intervention components, the number and difficulty of behaviors required to deliver the intervention, the number of groups or organizational levels targeted, the number and variability of outcomes and the degree of tailoring of the intervention permitted [63]. This makes the development and evaluation of these kind of interventions challenging in different ways.

First, a good theoretical understanding is needed of how the intervention causes change, so that weak links in the causal chain can be identified and strengthened [63,64]. A logic model describing the resources, activities, anticipated outcomes and impact should therefore be created prior to model implementation to illustrate the overall program theory and support planning, monitoring and evaluation [65]. Yet, although some of the studies did refer to theoretical frameworks underpinning their intervention (e.g. the Chronic Care Model or Behavioral Change Model), only one study reported a logic model or refered to an implementation framework [47]. Also, contextual understanding can help to ensure that each part of an intervention appropriately fits the context [66], especially for integrated care initiatives [12]. Context is also an essential consideration when evaluating an intervention $[66,67]$, as the same intervention may not work 
equally in different settings [68]. This requires a contextual analysis of not only the micro-level, but also the meso-level of the organisations involved in integrated care and the macro-level of policy drivers that allow for integrated care models to grow in all aspects of the SELFIE framework [15]. Accordingly, implementing interventions in a real-life setting requires continuous tailoring to the intervention setting to be as effective as possible.

Second, process evaluations are essential to determine whether negative findings are due to intervention or implementation failure. Yet only five of the included studies reported process evaluations [48, 69-72], while one reported a brief implementation analysis [46]. Melis et al. (2008), for example, observed a change in intervention focus towards caregivers of patients with advanced dementia, and that adherence to given recommendations increased when the care plan was tailored to the patients' preferences [71]. Metzelthin et al. (2013) evaluated reach, fidelity, dose delivered/received and perceived barriers allowing them to give clear recommendations for future interventions, such as better identification of the target group, as well as more training on the job and opportunites to exchange among healthcare professionals [72]. Stijnen et al. (2015) indicated time constraints due to administrative work, team meetings and patient monitoring as one of the main implementation barriers. In response, health care providers did not adapt the intervention protocol but instead chose to target a selected group of older people who would most benefit from the visitation programme [69]. These examples illustrate that process evaluations are needed to identify feasibility and implementation problems as well as to understand which core components are key in achieving the observed impact, so they can guide future research and clinical practice [73].

Third, using a single primary outcome when evaluating complex interventions is not considered the best use of the data [63]. Measuring a range of outcomes to be able to pick up unintended consequences is rather recommended. With outcomes ranging from 1 to 13 per study, this recommendation was largely taken up. However, the range of outcomes complicated pooling the results of the individual studies, especially because the same outcome, e.g., ADL, was operationalized in different ways. Both a common understanding of expected patient, provider and service outcomes for integrated care models to be used in this kind of research should be promoted and a database with common data elements (CDEs) supporting the comparison between integrated care studies needs to be built in line with recommendations from the National Institute of Health [74]. The development of CDEs needs to consider not only distal outcomes such as hospital admission, ED visits and quality of life, as was done by the included studies, but also proximal outcomes, such as patient satisfaction, well-being and the capacity and ability of the health care providers to deliver the intervention, as they will subsequently affect long-term effectiveness [73].

In all, evaluating complex interventions is challenging and for that digging into the "the black box" of the change process and not solely focusing on effectiveness evaluation is key in further strengthening the evidence-base of integrated care. Embedding process evaluations, development of logic models, context analysis and determining CDEs of proximal and distal outcomes, which are some of the key elements of implementation science, for the evaluation of integrated care models should therefore be put high on the research agenda. This is line with the overall recommendations of Smit et al. (2018) who analysed nine proactive primary care programs for older people in the Netherlands [75]. 
While further strengthening the evidence base with solid and scientifically sound hybrid evaluationimplementation studies [76], existing frameworks on integrated care, such as the SELFIE framework and WHO guidelines, can guide the further optimalisation of integrated care models. We observed that the care models included many core components suggested in the SELFIE framework, especially in terms of service delivery, leadership, and workforce [15]. Individualized care planning, multidisciplinary teams, informal care giver involvement, and shared decision making were present in the majority of studies, although rarely described in much detail. On the other hand, hardly any components were reported in view of financing, although coverage and reimbursement of interventions are crucial to provide equal access to health care services, a precondition to be able to provide high quality of care and critical for the sustainability of the model [77]. Similarly, evidence-base technology or products to support intervention delivery were rarely mentioned. Yet technology can be used to enhance communication and care coordination between involved care providers, monitor chronic diseases and support self-management [78, 79]. Remote monitoring, for example, allows home care nurses to maintain patient contact in a continuous way by assessing needs, educating patients and supporting caregivers without the need to travel. It can also prevent ED visits and hospitalisations by allowing early interventions and can help shift care from the hospital to home resulting in reduced health care costs [80]. Systematic and proactive screening to identify patients at higher risk for, for example, functional dependence, hospitalisation, or frailty is also recommended as it supports identifying individuals that would benefit most from integrated care interventions. Although there is no international consensus on which screening tool to use, several have been developed and validated for the community care setting, such as the Groningen Frailty Indicator, the PRISMA-7 and the FRAIL [81, 82]. The majority of the included studies indeed focused on a more at risk subpopulation by defining study eligibility criteria or conducting population or individual risk screening prior to conducting a more in-depth geriatric assessment, but in only six models an evidence-based tool was used to do so. The SELFIE framework also suggests to promote self-management by educating on e.g., behavioural change techniques, coping strategies, navigation through the care system, and medication adherence support, [15] but none of the articles fully explained what kind of self-management activities were conducted.

Lastly, having a care coordinator to organize care for people with multimorbidity is considered a core element of integrated care. Studies have shown that nurses play a crucial role in providing and managing care for the individual, collaborating with family members and coordinating care between health care professionals in the community [83]. This role can be effectively undertaken by nurses or nurse practitioners, but obtaining the right skills and competencies to fulfill the role as central care coordinator is essential for the care model to work effectively [84, 85]. Yet, in the included studies limited information was given regarding the skills and experience of the nurses and the level of education varied from registered nurses to nurse practitioners and advanced practice nurses. Although an explorative metaregression was not conducted to determine the impact of the nursing education level on the studied outcomes, previous research does support to favor advanced practice nurses for these central roles [21]. Care provided by advanced practice nurses or teams including an advanced practice nurses is as least equal to care or care models provided by medical doctors regarding process and clinical outcomes [86]. 
In conclusion, while this study has shown that many components considered essential in integrated care are included in the nurse-led care models, it could not provide solid evidence regarding their impact on health outcomes of the older population based on 19 prospective (quasi-) experimental studies at considerable risk of bias. The absence of evidence-based implementation theories, multilevel context analyses, and process evaluations hinders the ability to determine whether these nonsignificant findings are due to intervention or implementation failure. Challenges in proving effectiveness of complex care interventions in the older population need to be facilitated by clear and strong hybrid evaluationimplementation study designs in a realistic setting, logic models or program theories describing the expected impact of the planned intervention, a common understanding of how integrated care models affect the system, service, patients, and providers, as well as clearly defined proximal and distal outcomes, and timeframes, in which they are measured.

\section{Funding}

This review is part of the overall INSPIRE project which has received funding from the Velux Stiftung, the Swiss National Science Foundation, and the European Union's Horizon 2020 research and Innovation programme under the Marie Skłodowska-Curie grant agreement No 812656 as part of the TRANS-SENIOR project (www.trans-senior.eu).

\section{Declaration of conflicting interest}

The authors declare that there is no conflict of interest.

\section{Author Statement}

Mieke Deschodt: Conceptualization, Methodology, Investigation, Writing - Review \& Editing, Supervision, Project administration, Funding acquisition

Gwen Laurent: Investigation, Writing - Original Draft

Lonne Cornelissen: Conceptualization, Investigation, Writing - Original Draft

Olivia Yip: Writing - Review \& Editing

Franziska Zúñiga: Writing - Review \& Editing

Kris Denhaerynck: Formal analysis, Investigation, Visualization

Matthias Briel: Writing - Review \& Editing, Supervision

Azra Karabegovic: Investigation, Writing - Original Draft

Sabina De Geest: Conceptualization, Writing - Review \& Editing, Supervision, Funding acquisition

\section{Acknowledgements}

We would like to thank the INSPIRE consortium for their contributions in given feedback on the overall design and methods of the review during the INSPIRE research meetings: Prof. Dr. Nicole Probst-Hensch, 
PD Dr. Penelope Vounatsou, Prof. Dr. Andreas Zeller, Prof. Dr. Matthias Schwenkglenks, Dr. Carlos Quinto, PD Dr. Eva Blozik, Prof. Dr. Leah Zullig, and Samuel Stenz. We thank Rachel Jenkins for assisting with the data extraction and several critical reviews of the manuscript. 


\section{References}

1. Palladino, R., et al., Multimorbidity And Health Outcomes In Older Adults In Ten European Health Systems, 2006-15. Health Aff (Millwood), 2019. 38(4): p. 613-623.

2. Barnett, K., et al., Epidemiology of multimorbidity and implications for health care, research, and medical education: a cross-sectional study. Lancet, 2012. 380(9836): p. 37-43.

3. Humphries, R., Integrated health and social care in England--Progress and prospects. Health Policy, 2015. 119(7): p. 856-9.

4. WHO, Global strategy on people centered integrated health care service. 2015: p. 50.

5. Stange, K.C., The problem of fragmentation and the need for integrative solutions. Ann Fam Med, 2009. 7(2): p. 100-3.

6. Frandsen, B.R., et al., Care fragmentation, quality, and costs among chronically ill patients. Am J Manag Care, 2015. 21(5): p. 355-62.

7. AARP, Providing More Long-term Support and Services at Home: Why It's Critical for Health Reform. 2009: AARP Public Policy Institute.

8. Rechel, B., et al., Ageing in the European Union. The Lancet, 2013. 381(9874): p. 1312-1322.

9. Wolff, J.L., B. Starfield, and G. Anderson, Prevalence, expenditures, and complications of multiple chronic conditions in the elderly. Arch Intern Med, 2002. 162(20): p. 2269-76.

10. WHO, Integrated care for older people: guidelines on community-level interventions to manage declines in intrinsic capacity. 2017: p. 60.

11. Charles, A., et al., A year of integrated care systems: Reviewing the journey so far. 2018: The King's Fund.

12. Shaw, S., R. Rosen, and B. Rumbold, An overview of integrated care in the NHS: What is integrated care? 2011: The Nuffield Trust.

13. NICE, Home care for older people. Quality Standard. 2016: National Institute for Health and Care Excellence. 42.

14. Craig, P., et al., Developing and evaluating complex interventions: the new Medical Research Council guidance. BMJ, 2008. 337: p. a1655.

15. Leijten, F.R.M., et al., The SELFIE framework for integrated care for multi-morbidity: Development and description. Health Policy, 2018. 122(1): p. 12-22.

16. WHO, Key components of a well functioning health system. 2010: p. 2.

17. Goodwin, N., et al., Providing integrated care for older people with complex needs: Lessons from seven international case studies 2014: The King's Fund. p. 28.

18. WHO. Nursing and midwifery: Fact sheet. 2018 [cited 2019 March 1st]; Available from: https://www.who.int/mediacentre/factsheets/nursing-midwifery/en/.

19. Nigel Crisp, L. and B.M. Watkins Of Tavistock, The triple impact of nursing. Int J Nurs Stud, 2018. 78: p. A3-a4.

20. WHO, Interprofessional Collaborative Practice in Primary Health Care: Nursing and Midwifery Perspectives. World Health Organization. 2013, Human Resources for Health Observer.

21. Maier, C., L. Aiken, and R. Busse, Nurses in advanced role in primary care: Policy levers for implementation, in OECD Health Working Papers. 2017, OECD Publishing: Paris.

22. Smith, S.M., et al., Interventions for improving outcomes in patients with multimorbidity in primary care and community settings. Cochrane Database Syst Rev, 2016. 3: p. CD006560.

23. Mullins, L., L.E. Skemp, and M.L. Maas, Community Models of Care: A Scoping Review. Journal of Gerontological Nursing, 2016. 42(12): p. 12-20.

24. Liberati, A., et al., The PRISMA statement for reporting systematic reviews and meta-analyses of studies that evaluate healthcare interventions: explanation and elaboration. Bmj, 2009. 339(jul21 1): p. b2700-b2700.

25. Sterne, J.A.C., et al., RoB 2: a revised tool for assessing risk of bias in randomised trials. BMJ, 2019. 366: p. 14898.

26. Sterne, J.A.C., et al., ROBINS-I: a tool for assessing risk of bias in non-randomised studies of interventions. Bmj-British Medical Journal, 2016. 355.

27. Wilson, D.B. Practical Meta-Analysis Effect Size Calculator. Available from: https://www.campbellcollaboration.org/escalc/html/EffectSizeCalculator-Home.php.

28. Boult, C., et al., The effect of guided care teams on the use of health services: results from a cluster-randomized controlled trial. Arch Intern Med, 2011. 171(5): p. 460-6.

29. Ruikes, F.G., et al., Multicomponent Program to Reduce Functional Decline in Frail Elderly People: A Cluster Controlled Trial. J Am Board Fam Med, 2016. 29(2): p. 209-17.

30. Counsell, S.R., et al., Geriatric care management for low-income seniors: a randomized controlled trial. Jama, 2007. 298(22): p. 2623-33. 
31. Bouman, A., et al., Effects of a home visiting program for older people with poor health status: a randomized, clinical trial in The Netherlands. J Am Geriatr Soc, 2008. 56(3): p. 397-404.

32. Dorr, D.A., et al., The effect of technology-supported, multidisease care management on the mortality and hospitalization of seniors. J Am Geriatr Soc, 2008. 56(12): p. 2195-202.

33. Gravelle, H., et al., Impact of case management (Evercare) on frail elderly patients: controlled before and after analysis of quantitative outcome data. BMJ, 2007. 334(7583): p. 31.

34. Hoogendijk, E.O., et al., Effectiveness of a Geriatric Care Model for frail older adults in primary care: Results from a stepped wedge cluster randomized trial. Eur J Intern Med, 2016. 28: p. 4351.

35. Imhof, L., et al., Effects of an advanced practice nurse in-home health consultation program for community-dwelling persons aged 80 and older. J Am Geriatr Soc, 2012. 60(12): p. 2223-31.

36. King, A.I.I., et al., The effect of a gerontology nurse specialist for high needs older people in the community on healthcare utilisation: a controlled before-after study. BMC Geriatr, 2018. 18(1): p. 22.

37. Stijnen, M., et al., Effectiveness of a nurse-led home visitation programme on health-related quality of life and disability among potentially frail community-dwelling older people in primary care: a longitudinal, quasi experimental study. 2015: p. 24.

38. Looman, W.M., et al., The effects of a pro-active integrated care intervention for frail communitydwelling older people: a quasi-experimental study with the GP-practice as single entry point. BMC Geriatr, 2016. 16: p. 43.

39. Looman, W.M., I.N. Fabbricotti, and R. Huijsman, The short-term effects of an integrated care model for the frail elderly on health, quality of life, health care use and satisfaction with care. Int $\mathrm{J}$ Integr Care, 2014. 14: p. e034.

40. Markle-Reid, M., et al., Health promotion for frail older home care clients. J Adv Nurs, 2006. 54(3): p. 381-95.

41. Melis, R.J., et al., A randomized study of a multidisciplinary program to intervene on geriatric syndromes in vulnerable older people who live at home (Dutch EASYcare Study). J Gerontol A Biol Sci Med Sci, 2008. 63(3): p. 283-90.

42. Metzelthin, S.F., et al., Effectiveness of interdisciplinary primary care approach to reduce disability in community dwelling frail older people: cluster randomised controlled trial. BMJ, 2013. 347: p. f5264.

43. Morales-Asencio, J.M., et al., Effectiveness of a nurse-led case management home care model in Primary Health Care. A quasi-experimental, controlled, multi-centre study. BMC Health Serv Res, 2008. 8: p. 193.

44. Newcomer, R., et al., Outcomes of preventive case management among high-risk elderly in three medical groups: a randomized clinical trial. Eval Health Prof, 2004. 27(4): p. 323-48.

45. Reckrey, J.M., et al., The team approach to home-based primary care: restructuring care to meet individual, program, and system needs. J Am Geriatr Soc, 2015. 63(2): p. 358-64.

46. Ryvicker, M., et al., Improving functional outcomes in home care patients: impact and challenges of disseminating a quality improvement initiative. J Healthc Qual, 2011. 33(5): p. 28-36.

47. Stijnen, M., Towards proactive care for potentially frail older people in general practice : Development, feasibility, and effectiveness of the [G]OLD preventive home visitation programme, in School for Public Health and Primary Care (CAPHRI). 2015, Maastricht University: Maastricht. p. 212.

48. Suijker, J.J., et al., Effects of Nurse-Led Multifactorial Care to Prevent Disability in CommunityLiving Older People: Cluster Randomized Trial. PLoS One, 2016. 11(7): p. e0158714.

49. Melis, R.J.F., et al., The design of the Dutch EASYcare study: a randomised controlled trial on the effectiveness of a problem-based community intervention model for frail elderly people [NCT00105378]. BMC Health Services Research, 2005. 5(1).

50. Ruikes, F.G., et al., The CareWell-primary care program: design of a cluster controlled trial and process evaluation of a complex intervention targeting community-dwelling frail elderly. BMC Fam Pract, 2012. 13: p. 115.

51. Metzelthin, S.F., et al., The reduction of disability in community-dwelling frail older people: design of a two-arm cluster randomized controlled trial. BMC Public Health, 2010. 10: p. 511.

52. Fabbricotti, I.N., et al., Integrated care for frail elderly compared to usual care: a study protocol of a quasi-experiment on the effects on the frail elderly, their caregivers, health professionals and health care costs. BMC Geriatr, 2013. 13: p. 31.

53. Suijker, J.J., et al., Comprehensive geriatric assessment, multifactorial interventions and nurseled care coordination to prevent functional decline in community-dwelling older persons: protocol of a cluster randomized trial. BMC Health Serv Res, 2012. 12: p. 85. 
54. Nicolaides-Bouman, A., et al., Effects of home visits by home nurses to elderly people with health problems: design of a randomised clinical trial in the Netherlands [ISRCTN92017183]. BMC Health Serv Res, 2004. 4(1): p. 35.

55. Counsell, S.R., et al., Geriatric Resources for Assessment and Care of Elders (GRACE): a new model of primary care for low-income seniors. J Am Geriatr Soc, 2006. 54(7): p. 1136-41.

56. Hopman, P., et al., Effectiveness of comprehensive care programs for patients with multiple chronic conditions or frailty: A systematic literature review. Health Policy, 2016. 120(7): p. 818-32.

57. Ploeg, J., et al., Effectiveness of preventive primary care outreach interventions aimed at older people: meta-analysis of randomized controlled trials. Can Fam Physician, 2005. 51: p. 1244-5.

58. Looman, W.M., R. Huijsman, and I.N. Fabbricotti, The (cost-)effectiveness of preventive, integrated care for community-dwelling frail older people: A systematic review. Health Soc Care Community, 2019. 27(1): p. 1-30.

59. Huss, A., et al., Multidimensional Geriatric Assessment: Back to the Future Multidimensional Preventive Home Visit Programs for Community-Dwelling Older Adults: A Systematic Review and Meta-Analysis of Randomized Controlled Trials. The Journals of Gerontology Series A, 2008. 63: p. 298-307.

60. Rijken, M., et al., Assigning a Prominent Role to "The Patient Experience" in Assessing the Quality of Integrated Care for Populations with Multiple Chronic Conditions. Int J Integr Care, 2019. 19(3): p. 19.

61. King, A.I.I., et al., Implementation of a gerontology nurse specialist role in primary health care: Health professional and older adult perspectives. J Clin Nurs, 2018. 27(3-4): p. 807-818.

62. Monsen, K. and J. Deblok, Buurtzorg Nederland. Am J Nurs, 2013. 113(8): p. 55-9.

63. Craig, P., et al., Developing and evaluating complex interventions: Following considerable development in the field since 2006, MRC and NIHR have jointly commissioned an update of this guidance to be published in 2019. 2019, Medical Research Council.

64. Birken, S.A., et al., Criteria for selecting implementation science theories and frameworks: results from an international survey. Implement Sci, 2017. 12(1): p. 124.

65. Funnell, S.C. and P.J. Rogers, Purposeful Program Theory. Effective Use of Theories of Change and Logic Models. 2011, San Francisco: Jossey-Bass.

66. Smit, L.C., et al., Unravelling complex primary-care programs to maintain independent living in older people: a systematic overview. Journal of Clinical Epidemiology, 2018. 96: p. 110-119.

67. Powell, B.J., et al., A refined compilation of implementation strategies: results from the Expert Recommendations for Implementing Change (ERIC) project. Implement Sci, 2015. 10: p. 21.

68. Damschroder, L.J., et al., Fostering implementation of health services research findings into practice: a consolidated framework for advancing implementation science. Implement Sci, 2009. 4: p. 50.

69. Stijnen, M.M., et al., Nurse-led home visitation programme to improve health-related quality of life and reduce disability among potentially frail community-dwelling older people in general practice: a theory-based process evaluation. BMC Fam Pract, 2014. 15: p. 173.

70. Nicolaides-Bouman, A., et al., Home visiting programme for older people with health problems: process evaluation. J Adv Nurs, 2007. 58(5): p. 425-35.

71. Melis, R.J.F., et al., Process evaluation of a trial evaluating a multidisciplinary nurse-led home visiting programme for vulnerable older people. Disability and rehabilitation, 2010. 32(11): p. 93746.

72. Metzelthin, S.F., et al., A nurse-led interdisciplinary primary care approach to prevent disability among community-dwelling frail older people: A large-scale process evaluation. International Journal of Nursing Studies, 2013. 50(9): p. 1184-1196.

73. Proctor, E., et al., Outcomes for Implementation Research: Conceptual Distinctions, Measurement Challenges, and Research Agenda. Administration and Policy in Mental Health and Mental Health Services Research, 2010. 38(2): p. 65-76.

74. Sheehan, J., et al., Improving the value of clinical research through the use of Common Data Elements. Clin Trials, 2016. 13(6): p. 671-676.

75. Smit, L.C., et al., Unravelling complex primary-care programs to maintain independent living in older people: a systematic overview. J Clin Epidemiol, 2018. 96: p. 110-119.

76. Curran, G.M., et al., Effectiveness-implementation hybrid designs: combining elements of clinical effectiveness and implementation research to enhance public health impact. Med Care, 2012. 50(3): p. 217-26.

77. Patient-centered care for older adults with multiple chronic conditions: a stepwise approach from the American Geriatrics Society: American Geriatrics Society Expert Panel on the Care of Older Adults with Multimorbidity. J Am Geriatr Soc, 2012. 60(10): p. 1957-68. 
78. Darkins, A., et al., Care Coordination/Home Telehealth: the systematic implementation of health informatics, home telehealth, and disease management to support the care of veteran patients with chronic conditions. Telemed J E Health, 2008. 14(10): p. 1118-26.

79. Hagglund, M., et al., Bridging the gap: a virtual health record for integrated home care. Int $\mathrm{J}$ Integr Care, 2007. 7: p. e26.

80. Coye, M.J., A. Haselkorn, and S. DeMello, Remote patient management: technology-enabled innovation and evolving business models for chronic disease care. Health Aff (Millwood), 2009. 28(1): p. 126-35.

81. Raîche, M., et al., User guide for the PRISMA-7 questionnaire to identify elderly people with severe loss of autonomy. In Integrated service delivery to ensure persons' functional autonomy., ed. R. Hébert, A. Tourigny, and M. Gagnon. Quebec: Edisem. 147-65.

82. Woo, J., et al., Frailty Screening in the Community Using the FRAIL Scale. J Am Med Dir Assoc, 2015. 16(5): p. 412-9.

83. WHO, Enhancing the role of community health nursing for universal health coverage. Human Resources for Health Observer, 2017(18): p. 32.

84. Boult, C., L. Karm, and C. Groves, Improving chronic care: the "guided care" model. Perm J, 2008. 12(1): p. 50-4.

85. Kuluski, K., et al., What is Important to Older People with Multimorbidity and Their Caregivers? Identifying Attributes of Person Centered Care from the User Perspective. Int J Integr Care, 2019. 19(3): p. 4.

86. Martinez-Gonzalez, N.A., et al., Task-Shifting From Physicians to Nurses in Primary Care and its Impact on Resource Utilization: A Systematic Review and Meta-Analysis of Randomized Controlled Trials. Med Care Res Rev, 2015. 72(4): p. 395-418. 